\title{
A Wireless Monitoring System for Pulse-oximetry Sensors
}

\author{
María J. Morón ${ }^{1}$, Eduardo Casilari ${ }^{1}$, Rafael Luque ${ }^{1}$, José A. Gázquez ${ }^{2}$ \\ ${ }^{1}$ Dpto. Tecnología Electrónica, University of Málaga, Spain \\ ecasilari@uma.es \\ ${ }^{2}$ Dpto. de Arquitectura de Computadores y Electrónica, University of Almería, Spain
}

\begin{abstract}
This paper presents a wireless medical monitoring system. The system permits to receive and process in a single concentrator node (e.g. a laptop or a simple handheld device) the pulse-oximetry signals from one ore several monitored patients without using any wired infrastructure. The system, which is based on a piconet of Bluetooth sensors, can retransmit the medical signals by WLAN and GPRS.
\end{abstract}

The paper describes the practical application scenarios in which this type of systems could be of great utility.

\section{Introduction}

The increase in the processing and integration capacity of electronic devices, as well as the advances of low power wireless communications has enabled the development of unwired intelligent sensors for a wide set of applications. One of the most promising application fields is the medical telemonitoring of patients.

The impact of wireless technology in healthcare is expected to be enormous. According to Deloitte Consulting, wireless deployment in healthcare should yield more than $\$ 12$ billion in productivity gains by 2006 [8]

Among the main benefits of the incorporation of mobile technologies to the medical care we can mention:

- Mobile solutions have been shown to help improve patient safety, decrease the risk of medical errors and increase physician productivity and efficiency [6].

- Wireless sensors enable the patients' freedom of movements (preventing them from being hindered by cables) and promote new ways of patient monitoring such as home monitoring. This clearly improves patients' quality of life. Missed days of school or work are reduced and health related restrictions on normal daily activities are minimized. Moreover, it allows the "service continuity" of the healthcare attention, as it defines a constant link to medical professionals who are able to assist in the disease management process. This relieves the uncertainties the patient and other family members caused by self management.

- Patient telemonitoring facilitates the extension of healthcare services to remote and sparsepopulated areas avoiding the need of expensive medical premises.

- Ease in the exchange of information between sanitary agents. With mobile technologies, physicians can access patient information in a quick, efficient and secure way from any location. Providing physicians and clinicians wireless access to patient information and medical references largely eliminates the need to locate and read through patient charts or search for lab results from other departments. Using wireless handheld devices, physicians can view patient history, medicines, labs, etc, as well as add new treatments and prescribe [8]. A higher efficiency in the information management is directly linked to important cost savings and a better utilisation of human resources.

- Telemonitoring also increments the medical presence in emergency scenarios and makes possible remote diagnosis.

In the ambit of patient telemonitoring, one of the main objectives is the development of medical monitors that substitute traditional wired probes by intelligent wireless sensors, able to detect and transmit medical alarms. Wireless sensors must accomplish 
several critical requirements related to small dimensions and weight, ergonomicity and low power consumption (or long battery life).

During the last five years, both the research community and the industry have devoted many works to this field so different wireless medical sensors have been developed and even commercialised.

Using wireless sensors with standard interfaces, the tasks of the medical monitor are reduced to the transmission and processing of the patients' signs so they can be placed in a general-purpose electronic device such as a laptop or a PDA, which should imply a cost reduction in healthcare equipment. Moreover, the autonomy of medical sensors allows the definition of a new type of network (the wireless Personal Area Network or PAN) with wider possibilities to define new services and applications.

One of the first studies that introduced the concept of PAN was [3]. This study proposed a PAN that integrated the information generated by different intelligent sensors. The wireless interface of the PAN utilised a 916 MHZ RF transceptor from Link Technologies which provided a bi-directional bit-rate of $33.6 \mathrm{Kbps}$ in a range of $50 \mathrm{~m}$.

The concept of a "wearable" medical monitor was already considered in [7] where a prototype of wearable ECG monitor is described.

The work in [1] introduces a large-scale ad-hoc sensor network which enables medical staff to keep track of mobile patients by means of $3 \mathrm{G}$ technologies.

Authors in [2] define the concept of Mobile Health (M-Health) as the integration of technologies of mobile computing and medical sensors with wireless communications in a system of sanitary assistance. The main purpose of a M-Health system would be to allow the medical staff to access the patient information independently of the location of both (patient and medical specialists).

An original telemonitoring system is presented in [4]. The system, intended to be used during the patient transfers in ambulances and other medical mobile units, contemplates the utilisation of a narrowband radio-modem to retransmit the vital signs captured by a commercial medical monitor. The deployed infrastructure, which avoids the problems of saturation of a public phone network such as GPRS, radiates the radio-modem signals to a set of radio repeaters which in turn retransmit to the hospital the signals via Internet.

European multi-national MobiHealth Project [11] defines an architecture that provides permanent monitoring of patients outside the hospital. The project utilises the paradigm of a "Body-Area Network" by connecting diverse wired sensors to a $3 \mathrm{G}$ enabled device that continuously conveys the patient's vital signs to a reception point in the hospital.

Similarly the Spanish Corporation ITDeusto presented [10] an experience in which the ECG signals of cardiopathy patients are remotely supervised from a medical centre by means of probes connected to a wearable GPRS transmitter.

More recently [5] has introduced a more ambitious project, CodeBlue, a wireless architecture designed in Harvard University for emergency medical care. The project integrates low-power, wireless vital sign sensors, PDAs, and PC-class systems.

This article shows a simple and cost-saving architecture that defines a monitoring network of pulse-oximetry sensors. The developed system, which is basically an open-source software that can be set on a PC or a PDA, allows to monitor the SPO2 signal and pulse rate from several patients simultaneously. In addition, the system considers a hybrid interface in which the signals can be retransmitted via GPRS or WLAN.

The rest of the work is structured as follows: section 2 describes the general objectives of the project and the different application scenarios. Section 3 revisits some concepts about pulse-oximetry while section 4 comments in more detail the developed prototype. Finally section 5 presents some conclusions and project's current status.

\section{General Architecture and Application Scenarios}

We propose a general architecture in which the patient is provided with one or diverse wearable wireless sensors. The signals from the sensors are captured in an Intelligent Concentrator Node (ICN) which could be situated in a handheld computer carried by medical technicians, physicians or nurses.

An ICN would process and visualise the information from the sensors but it also should be able to communicate with other ICNs and with a Central Information Server (CIS) located in medical premises and equipped with a wired Internet connection.

The long-range communication between the different ICNs and CIS should be preferably based on public mobile data services such as GPRS (or UMTS). On the contrary, the connection between ICNs could benefit from WLAN technology. In this sense the 
configuration of the nodes as a MANET (Mobile ad hoc network) would be preferred to a centralised architecture. MANETs would confer a great robustness to the system communications as any ICN could retransmit to the CIS the signals flowing from the others.

As it respects to the connection between the ICNs and the sensor, nowadays, the most appropriate technology for developing medical sensors is Bluetooth [9]. The main reasons for the election are:

- $\quad$ Bluetooth achieves enough bit rate (up to 433.9 $\mathrm{Kb} / \mathrm{s}$ ) to transmit any vital sign.

- The typical transmission ranges of Bluetooth $(10 \mathrm{~m}-100 \mathrm{~m})$ are adequate to transmit the signals from wireless sensors with battery and power restrictions.

- Bluetooth implements mechanisms of authentication and encryption, which are critical aspects in the transmission of bio-signs.

- When compared with other wireless technologies (especially 802.11) Bluetooth can provide a reasonable bandwidth connection at a better price and power savings. Battery life and replacement is another crucial aspect when defining a healthcare telemonitoring application.

As application scenarios for the proposed architecture we can mention the three following general cases:

- Remote patient home-monitoring

In this case the patient would wear a Body Area Network (BAN) including one or several medical sensors and a wearable ICN. The ICN should detect and communicate the medical alerts to the CIS through GPRS. The ICN, which could be built on a PDA but should appear as a closed "black box" to the patient, ought to be remotely programmable from the CIS. Additionally one of the sensors could consist in a Bluetooth GPS (which also could be embedded in the wearable ICN). This would permit the immediate patient's location in case of requiring medical intervention.

- Monitoring of multiple patients in an medical environment

In this scenario the monitored patients can move freely in a supervised environment (e.g.: a hospital, an old people's home...). Different ICNs could be distributed in diverse fixed position in the supervised area. This would prevent the patients from "wearing" the ICN, which would not require a removable battery to be powered. As the patients move, some kind of "hand off" process would occur so that the vital signs would be transmitted by the nearest NCI. As a consequence, NCI nodes should detect and alert to the CIS when the sensors enters or abandons their respective coverage zone. Thus, this architecture would provide a sensor location service indirectly.

An interesting variant of this scenario is the case in which patients are immobilised (e.g.: on a bed) and the NCI is a mobile handheld controlled by the specialist. In this situation, as the medical staffs approaches the patients they would receive and visualise the signals from the identified sensors.

- Monitoring of multiple patients in an emergency scenario

In a mass casualty event (provoked by natural catastrophes, terrorist attacks, accidents...) the most critical medical aspect is to decide the order in which the victims must be assisted depending on the seriousness of their wounds. A sensor network as that described in our proposal would clearly help to optimise the medical resources in these situations. Non specialised staff could distribute the sensors (e.g.: pulse-oximeters) among the injured persons while the field personnel would be constantly informed about the most serious cases by the concentrator nodes (ICN). Depending on the dimensions of the area to cover (and the number of patients), these nodes could self-organize as an 802.11 ad-hoc network. As it has been previously suggested, if a location mechanism such as GPS is incorporated, the system could automatically provide the geographic coordinates of the medical alerts that are taking place. On the other hand, medical decisions on surgical interventions could be made remotely from a hospital if the signals are transmitted for example via GPRS.

\section{Pulse-oximetry}

A pulse oximeter is a noninvasive sensor which provides a continuous estimation of arterial hemoglobin oxygen saturation as well as the pulse rate. These important measurements inform about the blood oxygenation and can be updated with each heartbeat.

The pulse oximeter consists of a transductor which employs two Light Emitting Diodes (LEDs) and a fotodetector diode. One of the LEDs emits red light (with a wavelength of $\lambda=660 \mathrm{~nm}$ ) while the other generates infrared light $(\lambda=940 \mathrm{~nm})$. The sensor is based on the fact that the colour of blood varies 
depending on the oxygen it contains. In particular the hemoglobin molecules reflect more red light when they are oxygenated than when reduced while its behaviour is the opposite when the light is infra-red. The oximeter shines two beams of light through a finger (or earlobe etc.) which are finally received in the photodetector. By comparing the light intensity that is received (not reflected by the finger) for each wavelength, the oximeter is able to derive the light that is being absorbed by the blood and, consequently, the oxygen saturation. Moreover, the heart rate can be estimated from the slight change in the colour provoked by a beat of the heart pushing arterial blood into the finger. [9]

Conventional pulse-oximetry probes (as other traditional medical sensors) are attached to the medical monitors by wires. This technology limits the movements of the patient who is normally obliged to stay in bed. Recently, different vendors such as Nonin [12], Numed [13] or Cardguard [14] have developed bluetooth pulse-oximeters. This type of sensors results in greater flexibility and freedom of movement for both the patient and the healthcare provider.

\section{Developed Prototype and results}

The developed prototype is based on a Bluetooth piconet that includes an Intelligent Concentrator Node and a set of commercial pulse-oximeters or signal emulators. The emulator software, which has been implemented for both PC and handset as PDAs, permits to simulate the behaviour of a oximeter by generating pre-recorded SPO2 signals via Bluetooth links.

Figure 1 illustrates the simple structure of the prototype. The ICN, which acts as the master of the piconet, simultaneously receives the signals from the sensors or the emulators (slave nodes). This information can be retransmitted to another neighbour node via WLAN (e.g.: another concentrator node in a pyramidal hierarchy) or directly to a remote reception node (e.g.: in a hospital) via GPRS.

For our test-bed we employed a Bluetooth pulseoximeter from Nonin [12] as that illustrated in Figure 2. Nonin's Model 4100 is a wrist-worn module which can be connected to any compatible Bluetoothenabled device. The patient module incorporates a class II Bluetooth radio with a range of approximately $100 \mathrm{~m}$. The sensor is powered with two AA batteries, lasting for about 120 hours under continuous use. The oximeters, which implement the serial port Bluetooth profile, generate 75 information frames per second. The transmitted information includes the heart-rate and the saturation level estimated both for every four or eight heart-beats. The frames also provide the information to represent the pleth (plethysmographic) waveform. Additionally, the signal alerts about different aspects of the sensor functionality such as the battery status or the probe disconnection.

As the handset the system utilises a Compaq iPAQ Pocket PC 3975. This PDA incorporates an Intel 400 $\mathrm{MHz}$ Xscale microprocessor with $48 \mathrm{MB}$ flash ROM and $64 \mathrm{Mb}$ SDRAM, as well as an embedded Bluetooth interface.

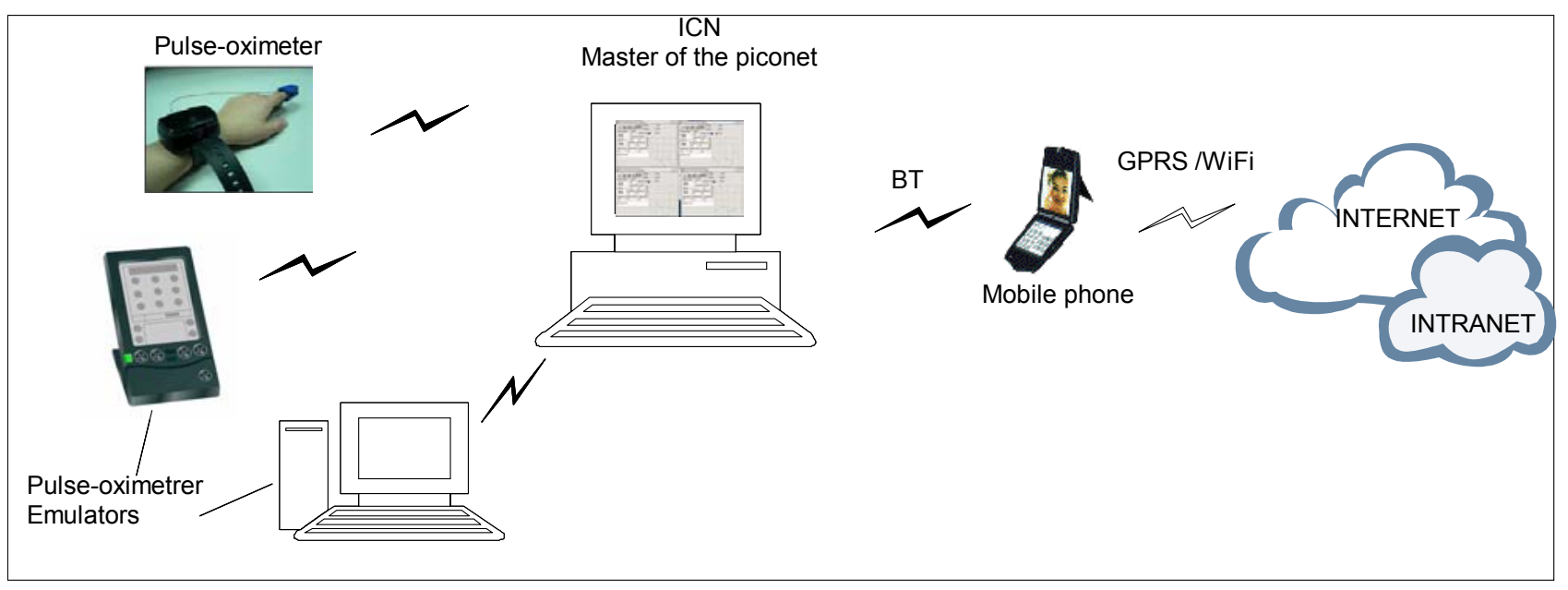

Figure 1. Prototype of the piconet 


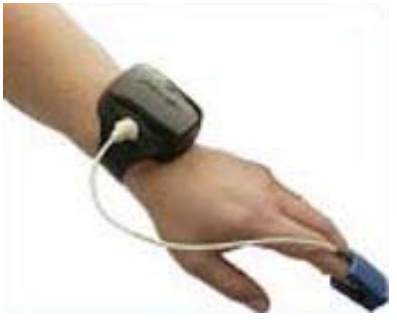

Figure 2. Employed SPO2 sensor

(Source: Nonin [12])

The software for the NCI and the emulators has been developed in two versions, one for PCs and other for PDAs. As the programming language $\mathrm{C}++$ was employed under a Linux environment. For the management of the Bluetooth communications and the definition of the graphical interface, the programs made use of the APIs Bluez (which implements upto RFCOMM in the Bluetooth stack architecture) and $g t k$ (for building the graphical user interface). After the debugging phase in the PCs, the software was incorporated to the PDAs by means of a crosscompilation tool.

The ICN node is continuously searching for the presence of new pulse-oximeters in its coverage area. As they are detected and an authentication phase is successful, the ICN opens a new connection with every new sensor. At that moment, the ICN begins to receive and decode the frame flow from the sensor showing the measurements (together with the status indicators and the possible medical alarms) in a monitoring panel as that of Figure 3.

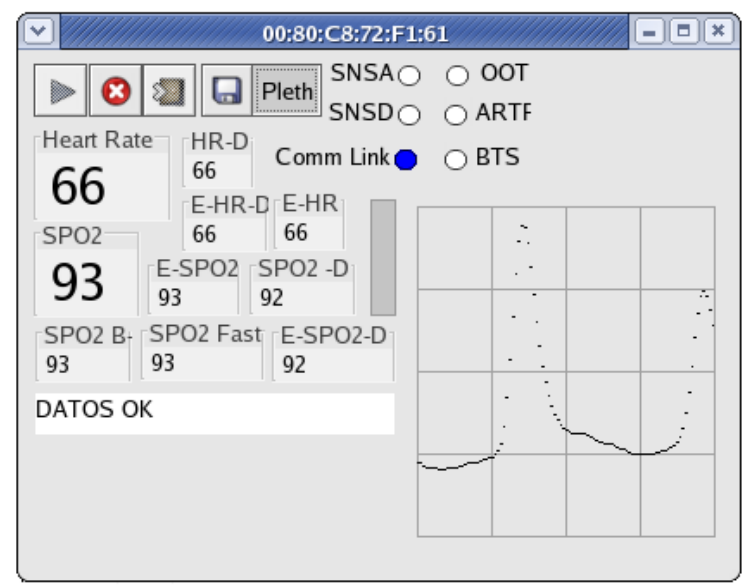

Figure 3. Monitoring panel
The developed software allows to save all the measurements in a file for further off-line analysis. Figure 4 includes a screenshot of the NCI graphical interface when it is run on a PDA. Figure 5 illustrates the software of the piconet master when it keeps track of four different signals.

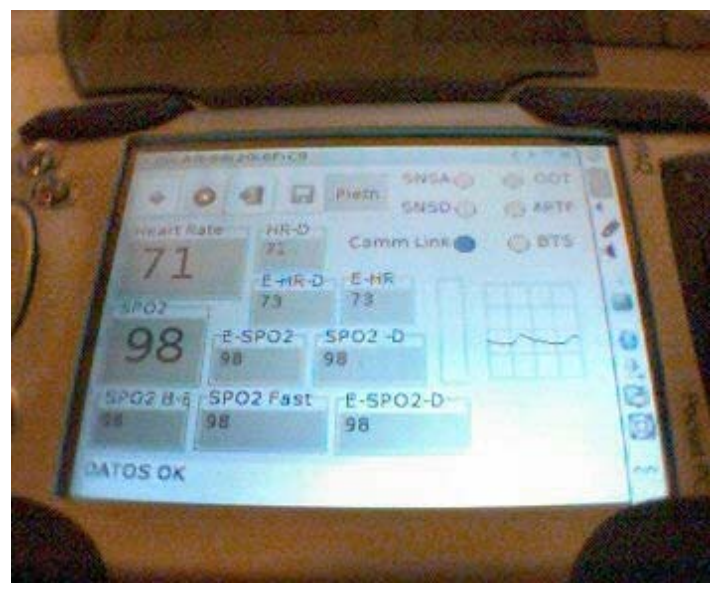

Figure 4. PDA Monitoring screen

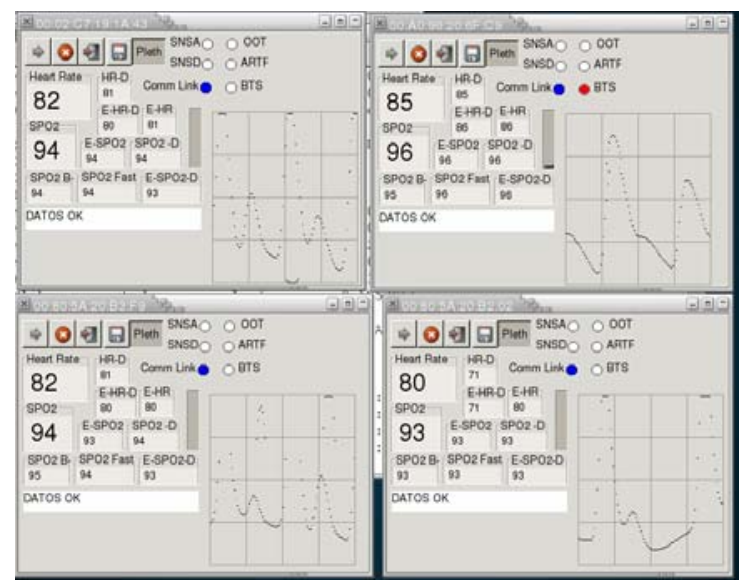

Figure 5. Monitoring screen for a piconet with four SPO2 sensors

\section{Conclusions: Current status and challenges}

This paper has presented a generic architecture for the management of medical wireless sensors. The developed prototype defines a network of Bluetooth pulse-oximetry sensors that are continuously monitored from concentrator nodes (ICNs). These 
ICNs, which could reside in a simple handheld (PDA), can inject the collected vital signs in Internet via GPRS or WLAN interfaces.

At the present moment, the prototype is still under development. Work in progress and future improvements include:

- Incorporation of GPS location to the monitored sensors or to the concentrator nodes (ICNs)

- Integration of other heterogeneous commercial sensors (e.g.: Bluetooth Blood pressure sensor, ECG probes,...)

- Definition of a MANET network that intercommunicates the diverse ICNs in a decentralised way.

- Simulation of the network for studying its performance in the case of managing an high number of sensors.

- $\quad$ Remote programming of the ICNs via Internet.

- A study to define redundant and hybrid communications (e.g.: GPRS/radio modem) that guarantees that the most critical signals are being properly received in the final reception node.

- Analysis of the feasibility of this type of applications in an actual scenario. In this sense we believe that wireless architecture should be initially considered not as an alternative to traditional wired monitoring system but as an added value service in healthcare assistance.

\section{Acknowledgements}

This work has been partially supported by Project No. TIC2003-07953-C02-01. We also want to thank doctors M. Noguerol and M. Lorente from Torrecardenas Hospital (Almeria, Spain) for their technical advice.

\section{References}

[1] F . Hu, J. Tillett, J. Ziobro and N.K. Sharma, "An energyefficient approach to securing tree-zone-based sensor networks", Proceedings of IEEE Global Telecomunications Conference (GLOBECOM 2003), vol. 3, no. 1, Dec. 2003, pp. 1430-1434.

[2] R.S.H. Istepanian, E. Jovanov and Y.T. Zhang, "Guest Editorial Introduction to the Special Section on M-Health: Beyond Seamless Mobility and Global Wireless Health-Care Connectivity", Proceedings of IEEE Transactions on Information Technology in Biomedicine, vol. 8, issue: 4 , Dec. 2004, pp. 405-414.

[3] E. Jovanov, J. Price, D. Raskovic, K. Kavi, T. Martin and R. Adhami, "Wireless Personal Area Networks in Telemedical Environment", Proceedings of the IEEE EMBS International Conference on Information Technology Applications in Biomedicine, 2000, pp.74-78.

[4] M. Noguerol, N.Novas, J.A. Gázquez, F.L Guillén, M. Lorente, and J. L. Blasco, "Wireless System of Communications Applied to Transporting of Critical Patiens", Proc. of $27^{\text {th }}$ World Congress in Internal Medicine, Granada (Spain), September 2004.

[5] D. Malan, T. Fulford-Jones, M. Welsh, and S. Moulton "CodeBlue: An Ad Hoc Sensor Network Infrastructure for Emergency Medical Care", Proc. of the MobiSys 2004 Workshop on Applications of Mobile Embedded Systems (WAMES 2004), Boston, MA, June, 2004.

[6] G. Malkary, Healthcare without bounds - trends in mobile computing, Spyglass Consulting Group, 2003.

[7] T. Martin, E. Jovanov and D. Raskovic, "Issues in Wearable Computing for Medical Monitoring Applications: A Case Study of a Wearable ECG Monitoring Device", Fourth International Symposium on Wearable Computers (ISWC'00), October, 2000, Atlanta (GA, USA)

[8] J. Park, "Healthcare Information Technology: Are You Plugged In?", document available at: http://www. surgicenteronline. com articles/341feat $1 . h t m l$.

[9] Promoter Members of Bluetooth SIG, Specification of the Bluetooth System - Version 1.1, February 2001.

[10] IT-Deusto: www.itdeusto.com

[11] MobiHealth Project, http://www.mobihealth.org/.

[12] Nonin Medical, Inc. http://www.nonin.com/

[13] Numed Holding, Ltd. http://www.numed.co.uk/

[14] Card Guard® AG, http://www.cardguard.com 